\title{
A NORMA REGULAMENTADORA 32 E SUAS IMPLICAÇÕES SOBRE OS TRABALHADORES DE ENFERMAGEM
}

\author{
Maria Lúcia do Carmo Cruz Robazzi ${ }^{1}$
} Maria Helena Palucci Marziale ${ }^{2}$

Robazzi MLCC, Marziale MH. A Norma Regulamentadora 32 e suas implicações sobre os trabalhadores de enfermagem. Rev Latino-am Enfermagem 2004 setembro-outubro; 12(5):834-6.

Os trabalhadores dos estabelecimentos de assistência à saúde muitas vezes enfrentam situações laborais inapropriadas não as considerando, entretanto, como perigosas, mesmo que as evidências científicas mostrem a presença de vários agentes de riscos ocupacionais nos ambientes de trabalho. O objetivo deste texto foi apresentar a Norma Regulamentadora (NR) 32, de Segurança e Saúde no Trabalho em Estabelecimentos de Assistência à Saúde. Esta NR é importante no cenário brasileiro, pois inexiste legislação específica que trate das questões de segurança e saúde no trabalho no setor da saúde e as normatizações existentes encontram-se esparsas e reunidas em outras NR e resoluções, que não foram construídas especificamente para tal finalidade. Conclui-se que mudanças benéficas poderão ser alcançadas por meio da implantação da referida NR, uma vez que procedimentos e medidas protetoras deverão ser realizadas, promovendo-se segurança no trabalho e prevenção de acidentes e doenças ocupacionais entre os trabalhadores da área da saúde.

DESCRITORES: riscos ocupacionais; acidentes de trabalho; doenças ocupacionais

\section{REGULATORY STANDARD 32 AND ITS IMPLICATIONS FOR NURSING WORKERS}

Workers in health care institutions often face inappropriate work situations without considering them as dangerous, however, even if scientific evidence demonstrates the presence of various occupational risk agents in work environments. This text aimed to present Regulatory Standard (RS) 32, about Safety and Health at Work in Health Care Institutions. This RS is important in the Brazilian scenario, due to the absence of federal legislation about work safety and health issues in the health sector and due to the fact that existing regulations are dispersed and joined with other RS and resolutions, which were not developed specifically for this purpose. It is concluded that the implantation of this RS may result in beneficial changes, since protection procedures and measures will have to be taken, promoting safety at work and occupational accident prevention among health workers.

DESCRIPTORS: occupational risks; accidents, occupational; occupational diseases

\section{LA NORMA REGLAMENTARIA 32 Y SUS IMPLICACIONES SOBRE LOS TRABAJ ADORES DE ENFERMERÍA}

Muchas veces, los trabajadores de los establecimientos de atención a la salud se enfrentan con situaciones laborales inadecuadas sin considerarlas, entretanto, como peligrosas, aún cuando las evidencias científicas muestran la presencia de varios agentes de riesgos ocupacionales en los ambientes de trabajo. La finalidad de este texto fue presentar la Norma Reglamentaria (NR) 32, de Seguridad y Salud en el Trabajo en Establecimientos de Atención a la Salud. Esta NR es importante en el escenario brasileño, ya que no existe legislación federal específica a cerca de las cuestiones de seguridad y salud en el trabajo en el sector de salud y las normatizaciones existentes se encuentran dispersadas y reunidas en otras NR y resoluciones, que no fueron construidas específicamente para tal finalidad. Fue concluido que podrán ser alcanzados cambios benéficos mediante la implantación de la referida $N R$, ya que procedimientos y medidas protectoras deberán ser realizadas, promoviéndose seguridad en el trabajo y prevención de accidentes y enfermedades ocupacionales entre los trabajadores del área de salud.

DESCRIPTORES: riesgos laborales; accidentes de trabajo; enfermedades ocupacionales

\footnotetext{
${ }^{1}$ Professor Titular, Enfermeira do Trabalho, Membro do Grupo Técnico que elaborou a NR 32, e-mail: avrmlccr@eerp.usp.br; ${ }^{2}$ Professor Associado, Enfermeira do Trabalho, e-mail: marziale@eerp.usp.br. Escola de Enfermagem de Ribeirão Preto, da Universidade de São Paulo, Centro Colaborador da OMS para o desenvolvimento da pesquisa em enfermagem
} 
Nos Estabelecimentos de Assistência à Saúde (EAS), há muitas ocasiões em que os membros da equipe de enfermagem enfrentam situações de risco no trabalho considerando-as, entretanto, corriqueiras, não Ihes dando a devida importância e pouco fazendo para que não se repitam. Evidências científicas têm mostrado que esses trabalhadores submetem-se aos vários agentes de riscos ocupacionais em seus ambientes de trabalho e nem sempre utilizam Equipamentos de Proteção Individual $(\mathrm{EPI})^{(1-4)}$.

São diversos os riscos ocupacionais a que estão submetidos os trabalhadores da área da saúde; entre eles encontram-se os riscos biológicos, os físicos, os químicos, os psicossociais e os ergonômicos ${ }^{(5-13)}$. Tais riscos predispõem os trabalhadores a se tornarem enfermos e a sofrerem acidentes de trabalho (AT) $)^{(1,4,10-11)}$, quando medidas de segurança não são adotadas.

No cotidiano do trabalho de enfermagem é comum observar-se situações de risco, tais como: administração de banhos no chuveiro nos pacientes, com os trabalhadores utilizando sacos de lixo amarrados nos pés, para protegê-los, devido à ausência de EPI apropriados; após a realização de coletas de sangue e outros líquidos corpóreos veiculadores de microrganismos patogênicos, muitos trabalhadores transportam seringas, agulhas $\mathrm{e}$ outros instrumentos pelos corredores dos EAS, sem proteção, até esses serem depositados em caixas de descarte, localizadas longe dos locais das coletas e muitas vezes com a capacidade esgotada, não sendo observados os limites estipulados pelo fabricante. Há a presença de trabalhadores com calçados abertos, bem como portando adornos os quais Ihes facilitam a possibilidade de contaminação. Além disso, sofrem quedas e conseqüentes AT por escorregarem em chãos lisos dos estabelecimentos de saúde; trabalhadores trajando uniformes desses EAS deslocam-se dos seus locais de trabalhos até os meios de transporte coletivos e daí para suas casas, sem ter tido oportunidades de mudar as suas roupas após a jornada laboral.

Situações dessa natureza foram detectadas pelo Grupo Técnico (GT) encarregado de elaborar o texto da nova Norma Regulamentadora (NR). Esta norma recebeu o número 32. A construção do texto dessa NR foi experiência ímpar, diante do pioneirismo da iniciativa, por parte do Ministério do Trabalho e Emprego do Brasil (MTE), atendendo às várias solicitações nesse sentido, das entidades que representam as diversas categorias de trabalhadores da saúde. Em 2002, a Escola de Enfermagem de Ribeirão Preto da Universidade de São Paulo (EERP-USP) foi chamada, através do GT, constituído de auditores fiscais do MET, para participar da construção dessa norma.

Desde as primeiras reuniões, para o GT, tornouse inequívoca a necessidade de se ter um texto normatizador oficial no País para a área da saúde, havendo consenso que essa era uma oportunidade importante e inquestionável de se regularizar as diversas e problemáticas questões que a envolvem. A partir daí, reuniuse material bibliográfico sobre os diversos problemas encontrados entre os trabalhadores da saúde. O GT passou a ter reuniões de trabalho e, após intenso e cuidadoso estudo, foi decidida a elaboração do texto da NR 32 por tipos de Riscos Ocupacionais e não por locais específicos dos estabelecimentos de saúde.

Para a elaboração dessa NR específica, foram utilizadas regulamentações da ANVISA (Agência Nacional de Vigilância Sanitária), dissertações de mestrado e teses de doutorado, recomendações e manuais já existentes no Ministério da Saúde, normas da Comissão Nacional de Energia Nuclear (CNEN), entre outras. Estudou-se também diretivas européias e americanas sobre o assunto, além das várias proposições de instituições importantes para a área, como as da Occupational Safety and Health Association - OSHA, aquelas do Centers for Disease Control - CDC, as da Organização Internacional do Trabalho e Organização Mundial da Saúde e as do National Institute of Occupational Safety and Health - NIOSH. À medida em que o texto ia sendo construído, consultou-se também alguns especialistas existentes no País, sobre determinados e específicos assuntos, bem como visitouse serviços instalados dentro de algumas EAS, onde foram entrevistados alguns trabalhadores com a finalidade de obter-se informações sobre a prática de como determinados procedimentos têm sido realizados nas diferentes regiões do país, buscando-se melhor entendêlos para normatizar-se sobre os mesmos, com maior propriedade.

A NR 32 é considerada de extrema importância no cenário brasileiro, como legislação federal específica que trate das questões de segurança e saúde no trabalho, no setor da saúde; as normatizações existentes encontram-se esparsas, reunidas em diversas outras NR e resoluções, que não foram construídas especificamente para tal finalidade. Acredita-se que mudanças benéficas 
poderão ser alcançadas por meio da referida normatização, uma vez que procedimentos e medidas protetoras deverão ser realizadas com vistas a promover segurança no trabalho e prevenção de acidentes e doenças ocupacionais.

A NR 32 encontra-se disponível no site www.trabalho.gov.br. Após a sua aprovação, os trabalhadores da área da saúde poderão contar com uma legislação específica para a sua proteção/segurança nos ambientes de trabalho dos EAS. É extremamente importante que todos os profissionais dessa área estejam informados sobre a referida legislação, bem como reivindiquem adequadas condições de trabalho, exercendo

\section{REFERÊNCIAS BIBLIOGRÁFICAS}

1. Bulhões I. Riscos do trabalho de enfermagem. $2^{\circ}$ ed. Rio de Janeiro (RJ): Editora Folha Carioca; 1994.

2. Robazzi MLCC, Marziale MHP. Alguns problemas ocupacionais decorrentes do trabalho de enfermagem no Brasil. Rev Bras Enfermagem 1999 jul/set; 52(3):331-8.

3. Silva VEF. Estudo sobre acidentes de trabalho ocorridos com trabalhadores de Enfermagem de um hospital de ensino. Ribeirão Preto. [dissertação]. Ribeirão Preto (SP): Escola de Enfermagem de Ribeirão Preto/USP; 1988.

4. Suazo SVV. Contribuição ao estudo sobre acidentes de trabalho que acometem as trabalhadoras de enfermagem em hospitais chilenos. [tese]. Ribeirão Preto (SP): Escola de Enfermagem de Ribeirão Preto/USP; 1999.

5. Marziale MHP. Condições ergonômicas da situação do pessoal de enfermagem em uma unidade de internação hospitalar. [tese]. Ribeirão Preto (SP): Escola de Enfermagem de Ribeirão Preto/USP; 1995.

6. Takeda E. Tuberculose: um estudo de sua situação entre pacientes internados e equipe de enfermagem de um hospital escola de Ribeirão Preto. [dissertação]. Ribeirão Preto (SP): Escola de Enfermagem de Ribeirão Preto/USP; 1996.

7. Rezende MP. Agravos à saúde de auxiliares de enfermagem resultantes da exposição ocupacional aos riscos físicos. [dissertação]. Ribeirão Preto (SP): Escola de Enfermagem de Ribeirão Preto/USP; 2003.

8. Xelegati R, Robazzi MLCC. Riscos químicos a que estão submetidos os trabalhadores de enfermagem: uma revisão de literatura. Rev Latino-Am Enfermagem 2003 maio/jun; 11(3):350-6.

9. Avendaño C, Grau P, Yus P. Riesgos para la salud de las enfermeras del sector publico de Chile. Rev Enfermaria 1995 dec; 30(102):15-26.

10. Benatti MCC. Acidente de trabalho em um hospital universitário: um estudo sobre ocorrência e os fatores de risco entre trabalhadores de enfermagem. [tese]. São Paulo (SP): Escola de Enfermagem/USP; 1997.

11. Miranda AF. Estresse ocupacional inimigo do enfermeiro?. [dissertação]. Ribeirão Preto (SP): Escola de Enfermagem de Ribeirão Preto/USP; 1998. seu papel enquanto trabalhadores e cidadãos, que cumprem com seus deveres e reafirmam seus direitos, principalmente aquele de trabalhar com segurança.

A NR 32 intitula-se Segurança e Saúde no Trabalho em Estabelecimentos de Assistência à Saúde e esteve em consulta pública durante aproximadamente oito meses e, atualmente, as sugestões estão sendo analisadas por equipe designada pelo MTE. Aguarda-se ansiosamente pelos resultados dos trabalhos e da aprovação legal do texto final, para que adequações sejam efetuadas nos postos de trabalho dos EAS de todo o Brasil.

12. Monteiro ABC. Biossegurança no preparo, administração e descarte de agentes antineoplásicos injetáveis pela equipe de enfermagem. [dissertação]. Ribeirão Preto (SP): Escola de Enfermagem de Ribeirão Preto/USP; 2001.

13. Marziale MHP, Kourrouski MFC, Robazzi MLCC. Riscos de acidentes no trabalho de enfermagem em centro cirúrgico. Rev Enfermagem UERJ 2000, jul/dez; 8(2):114-20. 